\title{
La sociología de la empresa en Francia: una construcción progresiva
}

\author{
Eguzki Urteaga \\ Universidad del País Vasco \\ eguzki.urteaga@ehu.es
}

Resumen: Este artículo analiza la sociología de la empresa que se desarrolla en Francia entre finales de la década de 1970 y principios de la década siguiente. Los primeros investigadores, que se enfrentaban a un vacío teórico, se interesaron en sus trabajos por los fenómenos identitarios y culturales, principalmente a través del estudio de las microculturas y de las sociabilidades colectivas. Todo ello ba desembocado en un intenso debate sobre el concepto de cultura de empresa, así como sobre la distinción entre la cultura obrera y la cultura empresarial. Estas aportaciones forman parte de un paradigma dominante encarnado por el enfoque institucional, cuyos principales representantes son Sainsaulieu y Segrestin.

Palabras clave: sociología, empresa, aportaciones, limites, Francia.

Abstract: This article analyzes the sociology of enterprise in France that develops from the end of the 1970's and the beginning of the 1980's. The first investigators face a theoretical emptiness, and their works are interested in the identity and cultural phenomena, with the study of micro-cultures and collective sociableness. All this has ended on an intense debate about the concept of culture of enterprise as well as on the distinction between the working culture and the enterprise culture. These contributions form a part of a dominant paradigm personified by the institutional approach which principal figures are Sainsaulieu and Segrestin.

Key words: sociology, company, contributions, limits, France. 


\section{Introducción}

Las reflexiones más novedosas de la sociología de las organizaciones, inspiradas en la obra de Michel Crozier y Erhard Friedberg(1977), tienen que ver, principalmente, con las administraciones públicas, pues las empresas industriales no han sido objeto de un desarrollo específico. Además, hasta finales de la década de 1970, la empresa no tenía muy buena prensa en Francia. A pesar de ser objeto de estudio (Sainsaulieu, 1992), era, como subraya Annie Borzeix (1986), una especie de decorado en cuyo seno se observaban las mutaciones tecnológicas, las estrategias patronales y sindicales, los cambios en la organización del trabajo, las relaciones de poder, etc. En definitiva, no constituía, como tal, un objeto de estudio.

Esa tendencia se invirtió a lo largo de los años ochenta, que coinciden en Francia con la rehabilitación social y política de la empresa. Como es lógico, la sociología no podía permanecer impasible frente a ese fenómeno y debía esforzarse por dar cuenta de él. Como resultado, aparece un creciente número de investigaciones centradas en la empresa y, paralelamente, se vislumbra, en torno a investigadores como Renaud Sainsaulieu y Denis Segrestin (1986), un intento de constituir una sociología de la empresa, a medio camino entre la sociología de las organizaciones y la sociología del trabajo. Su ambición es repensar la relación que une a empresa y sociedad. Aunque las investigaciones siguen siendo aisladas y carecen de coordinación, comparten el hecho de conceder especial atención a los fenómenos identitarios y culturales en el ámbito empresarial. No en vano, sus trabajos se enfrentan al vacío teórico que ha caracterizado durante un largo periodo la reflexión sobre la empresa.

\section{Un vacío teórico}

¿Cómo se puede explicar el escaso interés que despertó la empresa entre los sociólogos hasta la década de 1980? Borzeix alude a las divisiones internas propias de la disciplina sociológica: la sociología del trabajo y la sociología de las organizaciones, que han abordado la cuestión de la empresa, se desarrollan de manera autónoma en torno a referencias y problemáticas propias. Esta separación de los campos científicos se acompaña de una oposición ideológica. Así, la sociología del trabajo, impregnada por el análisis marxista, sigue estando vinculada al estudio del movimiento obrero y de los conflictos laborales, y accede a la empresa, habitualmente, por mediación de los sindicatos y de los representantes del personal. Por su parte, la sociología de las organizaciones, marcada por los trabajos anglosajones, se centra en las relaciones de poder y las instancias dirigentes y penetra en la empresa a través de ellas (Mathieu, 1986). 
Pese a la riqueza de sus producciones, estos dos ámbitos jamás han puesto la empresa en el centro de su reflexión.

\subsection{La sociología del trabajo y de la empresa}

Marc Maurice (1992) sugiere que la empresa se ha disuelto entre una sociología del trabajo orientada al análisis de situaciones laborales de los obreros en el seno del taller y una sociología de la sociedad industrial y del sistema de producción que la sostiene. Dicho de otra forma, el vacío teórico que caracterizaba la reflexión sobre la empresa sería resultado, en parte, de esta polarización entre una microsociología de la evolución del trabajo, básicamente empírica, y un enfoque macrosociológico de la sociedad capitalista industrial, fundamentalmente teórico. Ambas corrientes se han desarrollado sin apenas relación, pese a no ser incompatibles, pues los microanálisis de las situaciones de trabajo podían interpretarse, desde una perspectiva marxista, como la actualización de las fuerzas y contradicciones que atraviesan el sistema de producción capitalista.

No en vano, ninguno de los dos enfoques deja espacio para el análisis de este nivel intermedio que constituye la empresa. Marc Maurice observa precisamente que semejante sociología de la empresa habría podido permitir superar las lagunas y los límites de estas dos perspectivas, para así crear puentes entre la perspectiva macrosociológica teórica y el análisis empírico de las situaciones de trabajo que, habitualmente, carecen de una base teórica suficiente.

\subsection{La sociología de las organizaciones y de la empresa}

La sociología de las organizaciones, que apareció en Francia a finales de los años cincuenta de la mano de Michel Crozier, no ha colmado esa laguna. Si la comprensión de las relaciones sociales tanto en la empresa como en la administración se ha enriquecido, la entidad «empresa», por el contrario, tiende a disolverse en el análisis de los juegos de poder, que constituyen el objeto privilegiado de la sociología de las organizaciones. La empresa, pues, no consigue (Maurice, 1992) dotarse de una identidad en el espacio conceptual de la sociología de las organizaciones. Si puede ser el lugar de aparición de los sistemas de acción concretos, no aparece jamás como el actor que engloba o estructura semejantes juegos. El desplazamiento del análisis de la organización formal hacia el de la acción organizada, al que contribuye el análisis estratégico, contribuye a esta retirada teórica de la empresa.

Estas dos áreas temáticas de la sociología comparten su interés por lo que sucede en el seno de la empresa, sin por ello convertirla en paso obligado de su reflexión. No obstante, en la conjunción de la sociología del trabajo y de la sociología de las organiza- 
ciones, diversos trabajos se han interesado por los fenómenos identitarios y culturales en el seno de las organizaciones en general y de las empresas en particular.

\section{Los fenómenos identitarios y culturales}

Tres vías distintas, que se entrecruzan solo parcialmente, caracterizan este enfoque centrado en los fenómenos identitarios y culturales. La primera, que ocupa sobre todo a sociólogos e historiadores del trabajo y de las profesiones, se interesa por las microculturas de taller, las subculturas profesionales y, en general, por las formas de sociabilidad colectiva que se desarrollan en la empresa. La segunda vía, abierta por Renaud Sainsaulieu (1985), es más ambiciosa, pues confiere al trabajo y a la empresa un lugar central en la producción identitaria y cultural. La tercera y última, encarnada por Philippe d'Iribarne (1989; 1992), desarrolla la hipótesis de un modelo francés de empresa enraizado en la tradición cultural nacional preindustrial y sostenido por una lógica del honor.

\subsection{Microculturas y sociabilidades colectivas}

Numerosos sociólogos e historiadores del trabajo han puesto de manifiesto la existencia de sociabilidades colectivas, de comunidades profesionales y de micro y subculturas, elementos que constituyen la base de la vida laboral y de las relaciones laborales tanto en los talleres como en las oficinas y con los que la empresa debe contar para organizar su proceso productivo.

Existen diversos estudios sobre las comunidades profesionales que siguen la senda abierta por el análisis histórico de Georges Ribeill (1984) sobre los agentes ferroviarios. Ribeill muestra cómo, poco a poco, con el paso del tiempo, se constituye un cuerpo profesional resultado de la solidaridad entre los agentes de la explotación y el mantenimiento de las vías, que deben hacer frente a problemas de seguridad y regulación de los trenes. Esa identidad colectiva se ve reforzada por el modo de contratación, fomentado en el seno del entorno familiar de los agentes, por unas vías de ascenso específicas, el trabajo en equipo, una política de vivienda, etc. Esta comunidad profesional, dotada de una fuerte identidad colectiva, se enfrentará a los múltiples cambios emprendidos por la SNCF (electrificación de las líneas, lanzamiento del TGV, gestión informatizada, etc.) y desempeñará un papel crucial en la actividad sindical, especialmente en la convocatoria de huelgas, incluso cuando la unidad del grupo y su identidad se hallan en proceso de construcción (Corcuff, 1991). 
Pierre Bouvier (1989), que ha llevado a cabo diversas investigaciones sobre las comunidades de trabajo en los metros parisino y neoyorquino, desarrolla, desde un punto de vista socioantropológico, una perspectiva próxima. Así, en sus trabajos intenta describir los rituales profesionales, las sociabilidades colectivas y las normas integradoras que establecen los representantes y estructuran los colectivos de trabajo. Muestra que, en la RATP, esas prácticas y representaciones socioprofesionales, que constituyen la parte más importante de la historia del metro parisino, perduran más allá de la revolución tecnológica de los años setenta, cuando se asiste a la informatización de las diferentes operaciones del sistema de transportes.

Otros trabajos se han interesado, sobre todo, por los microgrupos que se forman en el seno de los talleres y otros ámbitos de trabajo. Así, llevando a cabo una experiencia de investigación-acción en una pequeña empresa de la metalurgia, compuesta por obreros especializados, obreros cualificados y braceros, Michel Liu (1981) constata la existencia de una pluralidad de microculturas de talleres que aparecen en el marco de las obligaciones fijadas por la tecnología y la organización del trabajo. Estas microculturas contribuyen a la definición de las funciones, así como a los ritmos y cantidades aceptables de trabajo; regulan las relaciones jerárquicas y las relaciones entre compañeros, y delimitan unos territorios colectivos y unos espacios individuales en el seno del taller. Por otra parte, tienen un rol a la vez instrumental (permiten la realización de la tarea que conviene realizar) y estructural, en la medida en que pretenden asegurar la continuidad del funcionamiento grupal más allá de las variaciones de los miembros que los componen. Por su parte, Emmanuelle Reynaud (1982), cuyas investigaciones se refieren a unos obreros de talleres de fabricación y a unos empleados de grandes almacenes, estudia igualmente el rol de estas microculturas en la constitución de identidades colectivas, así como en la capacidad movilizadora y el ardor en la lucha.

A pesar de su interés, semejantes orientaciones siguen quedando confinadas a un análisis estrecho de las relaciones sociales de trabajo. De este modo, si bien dan cuenta de fenómenos culturales que se desarrollan en el seno de la empresa, no ponen a esta última en el centro de su reflexión.

\subsection{La empresa como espacio de producción identitaria y cultural}

En L'identité au travail, Renaud Sainsaulieu (1985) pone de manifiesto la importancia que tienen los procesos culturales en el seno de las regulaciones sociales internas a la empresa. La obra se fundamenta en unas investigaciones empíricas llevadas a cabo en varias empresas y tiene por objetivo cuestionar y completar los logros de la sociología del trabajo, de la sociología de las organizaciones y de la psicología social. Una de las 
ideas destacadas es que las relaciones de trabajo no están aisladas del marco en que se expresan y que les confiere cierto sentido, es decir, la empresa.

El último capítulo, titulado «la acción cultural del trabajo organizado», defiende la tesis de que la experiencia diaria de las relaciones sociales es tal en las organizaciones contemporáneas que no se limita a las normas de comportamiento presentes en los lugares de trabajo. Según Sainsaulieu, alimenta unas representaciones colectivas y unos valores comunes que la superan y orienta las personalidades individuales en sus elecciones y valoraciones. En estos desfases entre la experiencia adquirida anteriormente y la experiencia inmediata de relaciones conflictivas en el seno de la empresa se reactivan y renuevan los sistemas de valores culturales de los actores sociales.

En definitiva, la familia y el sistema educativo han dejado de tener el monopolio de la socialización y de la transmisión de los valores culturales. Renaud Sainsaulieu muestra con fuerza que las relaciones de trabajo y el lugar donde se ejercen (la empresa) estructuran la identidad individual y colectiva. El trabajo en la empresa se convierte, en las sociedades industriales contemporáneas, en un nuevo lugar de producción y de aprendizaje cultural, como en otros tiempos lo fueron el universo de las corporaciones, la familia burguesa o la Iglesia católica. Sin embargo, la empresa y las relaciones de trabajo no se estructuran de la misma forma, debido a la historia y las tradiciones preindustriales de la sociedad en que se ubican.

\subsection{El modelo francés de empresa}

Philippe d'Iribarne (1989) pone el énfasis en los caracteres nacionales de los modos de gestión de las empresas. En realidad, la idea no es completamente nueva. Marc Maurice, François Sellier y Jean-Jacques Silvestre (1982) ya mostraron, a partir de un estudio comparativo entre Francia y Alemania, que las estructuras internas de las empresas dependen de sus entornos educativos, sindicales y políticos. Asimismo, Crozier (1964) había defendido la tesis de que el fenómeno burocrático era un «fenómeno cultural francés» que afectaba no solo a la administración pública, sino también a las empresas industriales.

D'Iribarne se desmarca de sus predecesores, especialmente de la tesis de Crozier, al considerar que esta no indaga suficientemente en la historia y las tradiciones culturales, lo que explica la especificidad de las empresas francesas. Si estas últimas están marcadas, a imagen de la administración, por una fuerte estratificación de los grupos profesionales, D'Iribarne no ha encontrado, a lo largo de sus observaciones, las reglamentaciones impersonales, el temor de la relación de cara a cara o la centralización mencionados por Crozier. En su opinión, lo que caracteriza a las empresas francesas y las distingue de sus homólogas holandesas o americanas es el vigor de los particularis- 
mos y de las tradiciones de oficios fundados en unas finas distinciones entre actividades más o menos nobles, como las que existían en el Antiguo Régimen. Les corresponde una lógica del honor que continúa rigiendo, en las empresas contemporáneas, tanto las relaciones jerárquicas como las relaciones entre grupos profesionales.

Sometidas a crecientes exigencias en materia de cooperación interna, fruto de las presiones derivadas de las necesidades económicas, las empresas se ven obligadas a concebir una manera de reinterpretar las tradiciones de enfrentamiento y de cooperación de la sociedad en cuyo seno se han implantado. Así, Philippe d'Iribarne da cuenta de las diferentes vías utilizadas por la modernidad industrial para, al mismo tiempo, respetar las tradiciones. No en vano, el debate contemporáneo sobre la dimensión cultural de las empresas se orienta igualmente hacia el análisis de las estructuras culturales propias de cada empresa. Conviene estudiar detenidamente esta perspectiva centrada en la noción de cultura empresarial.

\section{La noción de cultura de empresa}

Para Renaud Sainsaulieu (1987), la noción de cultura de empresa, tal y como la utilizan las ciencias de gestión y, en cierta medida, las ciencias sociales, se acompaña de cierto número de problemas teóricos que resultan de las hipótesis en que se fundamentan. Sainsaulieu identifica cinco hipótesis principales y hace referencia, para cada una de ellas, a las cuestiones que siguen sin responder o los puntos que aún no han sido resueltos.

La primera hipótesis afirma la existencia de una cultura común, formada por representaciones y valores y compartida por todos los miembros de la organización. Semejante afirmación no constituye ninguna evidencia, pues contradice la representación habitual de la empresa en términos de conflictos sociales y antagonismos de clase. La naturaleza del trabajo, las diferencias salariales y las afiliaciones sindicales, entre otros aspectos, son elementos que generan fuertes tensiones en el seno de la empresa y que tienden a relativizar la existencia de intereses compartidos, así como de una cultura común. Pierre-Eric Tixier (1989) completa sus críticas indicando que hemos pasado de una visión vertical de las relaciones sociales, en términos de clases sociales opuestas, a una visión horizontal que representa la empresa como una comunidad de individuos diferenciados. Mientras que la primera infravaloraba todo lo que podía constituir la pertenencia común a una misma organización, la segunda sobrevalora los elementos de consenso.

La segunda hipótesis en que se fundamenta la noción de cultura de empresa pone énfasis en el hecho de que los juegos y las estrategias de los actores en el seno de las 
organizaciones están alimentados por una serie de valores interiorizados y sistemas de representación. Esta idea (Sainsaulieu, 1987) consagra una ruptura formal con la sociología de las organizaciones inspirada en Crozier, para la cual las estrategias de los actores tienden a ser exclusivamente interpretadas en términos de oportunidades de poder. Friedberg (1993) mantiene una posición más matizada, pues precisa que los comportamientos de los actores dependen igualmente de su historia personal y de los procesos de aprendizaje que ha llevado a cabo, los cuales condicionan tanto su percepción de la situación como su capacidad para ajustarse a ella. La cuestión que se plantea entonces es la del peso de estas representaciones culturalmente transmitidas en los juegos y en las estrategias elaboradas por los actores.

La tercera hipótesis contemplada por el concepto de cultura de empresa es la que la asocia a un proyecto movilizador. La empresa, desde esta perspectiva, no se limitaría a un espacio donde se manifestarían unas prácticas colectivas heredadas del pasado, de los valores y de los rituales compartidos, sino que sería una identidad específica, que movilizaría igualmente a sus miembros en torno a un proyecto civilizador. ¿Cómo pasar de unas representaciones y prácticas compartidas entre los miembros de una misma comunidad a la adhesión y el compromiso en torno a un proyecto que implica unos objetivos precisos que alcanzar, unas realizaciones que concretar $y$, además, aunar los comportamientos individuales? Tixier (1988), al hilo de esta reflexión, insiste en la fragilidad de los instrumentos de gestión, fragilidad que resulta del carácter a menudo efímero de las referencias simbólicas que movilizan. Insiste en los riesgos de desfase entre la imagen dada por el proyecto de empresa y la realidad tal y como es vivida por el mayor número de personas, que puede derivar en que la adhesión sea sustituida por la desmotivación.

La noción de cultura de empresa implica una cuarta hipótesis, que percibe la existencia de una microsociedad capaz de instaurar de forma duradera las reglas de su funcionamiento social interno. Según Liu (1981), esta hipótesis impone la imagen de una unidad social que cuenta con una estructura y unos rasgos originales relativamente estables. El problema que resulta de esta hipótesis estriba en la tendencia a considerar la empresa como un universo cerrado relativamente distinto de su entorno social exterior.

Una quinta hipótesis, que en cierta medida se opone a la anterior, se asocia a veces con la utilización de la noción de cultura de empresa. Desarrollada por Sainsaulieu y Segrestin (1986), sugiere que la empresa es productora de sociabilidades, identidades y valores susceptibles de incidir en la sociedad global y de influir en ella. Esta hipótesis, además de suponer unas formas de aprendizaje, transmisión y difusión de estos fermentos culturales en dirección de la sociedad, nos lleva a preguntarnos sobre la compatibilidad entre estos mecanismos y las exigencias más triviales de la producción. 
El análisis de esta noción de cultura de empresa genera más preguntas que respuestas. Su carácter operativo solo se puede afirmar por medio de estudios empíricos que pueden cuestionar ciertas ideas preconcebidas.

\subsection{Cultura obrera y cultura empresarial}

Generalmente se admite que el lugar ocupado por la empresa a lo largo de los años ochenta es resultado del debilitamiento de los sistemas de identificación anteriores. Maryse Tripier (1986), preguntándose sobre las formas de elaboración de la cultura empresarial, parte de un planteamiento prácticamente inverso. $\mathrm{Si}$ es preciso realizar numerosos esfuerzos y si tantos profesionales de los recursos humanos son necesarios para ayudar a construir una cultura de empresa, quizá es porque aún siguen estando presentes otros sistemas de identificación: los valores del oficio, pero también la pertenencia étnica, regional o sindical, que continúan teniendo sentido para los trabajadores. Simultáneamente, las empresas son también productoras de modelos culturales, sobre todo cuando mantienen unas relaciones de larga duración con sus asalariados o su entorno local. Por lo tanto, ambos aspectos coexisten en el seno de las empresas, de modo que los comportamientos de los miembros dan cuenta de una yuxtaposición más o menos jerarquizada de sistemas de identificación diferentes, internos y externos a la empresa. Tripier, precisamente, intenta analizar esta imbricación entre unos distintos sistemas de identificación.

Sus investigaciones tienen por objeto una empresa metalúrgica de los años ochenta, subcontratada en el sector de la automoción. Esta empresa, que empleaba a cerca de mil trabajadores en 1979, quebró en 1985 y dejó a más de 500 personas sin trabajo. Se trata de una comunidad industrial relativamente aislada, inscrita en una sociedad local rural estructurada por la existencia de una central sindical única y poderosa: la CGT. Esta comunidad se caracteriza por la existencia de una fuerte cultura obrera, proletaria, que se fundamenta en la articulación de dos dimensiones: por una parte, el sentimiento de pertenencia a una amplia comunidad opuesta a otros grupos sociales $y$, por otra, la reducción de esa representación al ámbito de la empresa, que se vive como un espacio cerrado de enfrentamientos y relaciones de fuerza.

Durante las crisis sociales que conoce la fábrica, se produce un paulatino desplazamiento de esta cultura obrera hacia la movilización identitaria basada en la empresa. La aproximación simbólica a la empresa por parte de su grupo obrero se traduce en movilizaciones del tipo «no toques a mi empresa» frente a la competencia alemana, cuya presencia amenaza el empleo, y en un rechazo de cualquier intervención exterior, tanto la del senador que se había proclamado mediador como la de la instancia regional de la CGT. De este modo, la oposición entre el «nosotros» y el «ellos» no se reduce a 
la oposición entre la dirección y los trabajadores, elemento estructurante de la cultura obrera, sino que designa también la oposición entre la empresa y lo que le es externo. Este reconocimiento de la empresa como entidad que tiene sentido y con la que cada uno puede identificarse como parte del grupo obrero se amplía, asimismo, a las otras categorías de trabajadores de la empresa, incluidos los cuadros directivos, y, posteriormente, al conjunto de la sociedad local.

No en vano, para que esta identificación se produzca, no bastan la situación de crisis y la amenaza de cierre. Supone también que las bases de la comunidad no sean artificiales. En el caso estudiado, es la comunidad obrera (por su carácter de enclave social, por su dilatada historia y por la continuidad intergeneracional) lo que constituye la matriz. Con este ejemplo, Tripier muestra que la cultura de empresa no se construye a partir de la nada, sino a partir de lo existente, de manera que cultura obrera y cultura de empresa no se oponen necesariamente. Además, Tripier pone énfasis en un elemento fundamental: la noción de cultura tal y como la construye no hace referencia a un proceso de integración o de identificación del que quedaría excluida cualquier realidad conflictiva.

\section{Un paradigma dominante}

Sainsaulieu y Segrestin pertenecen a dos tradiciones distintas: el primero sigue la senda de la sociología de las organizaciones inspirada en Crozier, mientras que el segundo ha desarrollado sus estudios en el ámbito de la sociología del trabajo, donde se ha especializado en el análisis del sindicalismo y el fenómeno corporativo. Ambos se reúnen, a mediados de la década de 1980, en un intento de pensar e interpretar la rehabilitación de la empresa en Francia, pues, en poco más de diez años, había pasado de ser prácticamente ignorada, descuidada e, incluso, despreciada a convertirse casi en objeto de culto. Sainsaulieu y Segrestin comparten la asunción de la multiplicidad de los discursos de valorización y, además, el rechazo en considerar ese interés como un simple fenómeno de moda. Lo que está en juego es la búsqueda de un nuevo estado de regulación de las relaciones sociales. Ambos elaboran una nueva perspectiva.

\subsection{La perspectiva institucional de la empresa}

El enfoque institucional de la empresa (Sainsaulieu y Segrestin, 1986), siguiendo la vía abierta por Maurice, Sellier y Silvestre (1982), intenta comprender el lugar que ocupa la empresa en la sociedad contemporánea. Al término de su investigación sobre las políticas educativas y la organización industrial en Francia y Alemania, los autores 
citados preconizaban un análisis societario de la empresa, concebida al mismo tiempo como organización autónoma y como institución construida en y por las fuerzas que se expresan en la realidad salarial. Segrestin y Sainsaulieu profundizan en esta vía y consideran que la empresa no es una organización más, sino una institución que ocupa un lugar central en la sociedad actual y que, por este motivo, es capaz de asegurar una nueva regulación de las relaciones sociales. Esta regulación se concreta, según estos autores, en tres niveles: el nivel identitario, el nivel cultural y el nivel del cambio.

Sainsaulieu y Segrestin observan que, en un contexto general de debilitamiento de las referencias sociales habituales, la empresa se afirma como vector de producción identitaria. Esta ha dejado de plantearse únicamente en términos de militancia, de comunidades profesionales o de grupos de pertenencia informales, y pasa a fundamentarse en la propia empresa. La empresa, de este modo, se convierte en un reto social que cuenta con un gran potencial de reconocimiento y de redefinición de las identidades colectivas, sin que por ello se pueda asimilar este fenómeno al tradicional «espíritu casero». Este último se caracterizaba por una identificación total con la comunidad, gracias a la homogeneidad de quienes pertenecían a la misma familia y a una articulación jerárquica muy rígida, algo que no ocurre en las formas actuales, más flexibles y menos proclives a la fusión. Estas evoluciones, además, no aparecen ex nibilo; por el contrario, se trata de redefiniciones identitarias que se fundamentan en lo existente.

La valorización de la empresa como objeto sociológico tiene que ver con la autonomía de su cultura y de su desarrollo. No obstante, esa imagen de la empresa como célula cohesionada gracias a una cultura común concebida como vínculo simbólico de valores compartidos (sentimientos de pertenencia, capacidad colectiva de producción, consenso en torno a proyectos, comunidad de trabajo, etc.), no se corresponde con la mayoría de los casos que se plantean. En efecto, en numerosas empresas, toda una serie de experimentos (círculos de calidad, grupos de expresión, descentralización, horarios flexibles, trabajo a domicilio) han dado lugar a unos procesos de autonomía y diferenciación cuya compatibilidad con la visión de una comunidad integrada está por demostrar. Estas experiencias parecen ser contradictorias con el objetivo de la integración, en la medida en que se traducen en una mayor diversidad y diferenciación. Las empresas contemporáneas no son ni tribus, ni familias, ni pueblos aislados (Sainsaulieu, 1987). Volver al concepto de cultura para comprender la dinámica social de las empresas supone que todos los fenómenos identitarios y culturales tienen la posibilidad de realizarse.

En el tercer nivel, el del cambio, se plantea la cuestión de las relaciones entre la empresa y la sociedad en su conjunto. La hipótesis del cambio defendida por Sainsaulieu y Segrestin se articula en torno a la siguiente idea: la empresa no es solo un lugar de producción y de difusión de reglas, sino que tiende a generar una serie de valores, modelos 
y sistemas de representación. El cambio resulta del hecho de que la empresa evoluciona en su relación con la sociedad y, por esta razón, puede contribuir o no al cambio de esta última. La dinámica de la relación entre empresa y sociedad es el factor de cambio: cambio en el seno de la empresa, pero también de la sociedad en su conjunto.

Así concebido, el proyecto de la sociología de la empresa es ambicioso y toma nota de la autonomía de la empresa, incluso cuando esta sigue siendo relativa y objeto de debates (Ramanantsoa, 1992). Tanto la perspectiva institucional, preconizada por Sainsaulieu y Segrestin, como el enfoque societario, al que se refiere Maurice, intentan precisamente pensar las relaciones entre la empresa y su entorno, y cuestionan la oposición entre el interior y el exterior de la empresa. Pero van más allá que la sociología de las organizaciones inspirada en Crozier, porque no se trata únicamente de poner el énfasis en las interrelaciones con el entorno inmediatamente pertinente, sino de ver en qué medida la «institución-empresa» es capaz de influir en el mundo que la rodea $y$, por lo tanto, de contribuir al cambio de la sociedad en su conjunto. En última instancia, desde esta perspectiva, la empresa deja de ser una simple organización para convertirse en institución.

Las limitaciones de esta perspectiva estriban en que sus orientaciones teóricas están estrechamente vinculadas al discurso de la rehabilitación social y política de la empresa en Francia, porque la promoción simbólica de la empresa en los años ochenta responde tanto a los éxitos del liberalismo económico como al debilitamiento de la lectura marxista de la sociedad. En este contexto intelectual, se corre el riesgo de presentar una visión exclusivamente consensual de la empresa y de dejar de lado las relaciones de dependencia y dominio que se dan en su seno entre los diversos grupos y actores.

\section{Conclusión}

En este artículo se ha analizado la sociología de la empresa que se desarrolla en Francia entre finales de la década de 1970 y principios de la década siguiente. Los primeros investigadores se enfrentaban a un vacío teórico, pues ni la sociología del trabajo ni la sociología de las organizaciones habían abordado la realidad empresarial como objeto de estudio propio. Esos autores se interesan por los fenómenos identitarios y culturales, a través del estudio de las microculturas y de las sociabilidades colectivas y preguntándose por la existencia o no de un modelo francés de empresa. Todo ello ha desembocado en un intenso debate sobre el concepto de cultura de empresa, así como sobre la distinción entre la cultura obrera y la cultura empresarial. Tales aportaciones forman parte de un paradigma dominante encarnado por el enfoque institucional, cuyos principales representantes son Sainsaulieu y Segrestin. 
Si la sociología de la empresa en Francia ha conocido un auge incuestionable y ha contribuido a mejorar el conocimiento del mundo empresarial, no es menos cierto que presenta algunas carencias. En este sentido, además de centrarse excesivamente en las dimensiones cultural e identitaria, ha sido incapaz de integrar las aportaciones de las investigaciones, tanto teóricas como empíricas, llevadas a cabo en otros países, así como de abrirse a nuevas perspectivas que, si bien no pertenecen con propiedad a la sociología de la empresa, del trabajo y de las organizaciones, pueden permitir renovar sus reflexiones. Así, por ejemplo, la noción de «montaje heterogéneo» desarrollada por Boltanski y Thévenot (1991) insiste en la exigencia de justificación de las organizaciones, así como en la pluralidad de los regímenes de acción. Por otra parte, la noción de campo desarrollada por Bourdieu (1980) permite concebir las empresas como sistemas estructurados y jerarquizados donde coexisten dominantes y dominados que luchan para la consecución de recursos económicos, culturales, sociales y simbólicos. Por último, la empresa se puede asimilar a una red (Lazeaga, 1994), lo que permite precisar el análisis estratégico y, al mismo tiempo, renovar el estudio de la innovación.

\section{Bibliografía}

Boltanski, L. y Thevenot, L. (1991). De la justification. Paris: Gallimard.

Borzeix, A. (1986). «Avant-propos». Sociologie du travail, 3: 231-235.

Bourdieu, P. (1980). Questions de sociologie. Paris; Minuit.

Bouvier, P. (1989). Le travail au quotidien. Paris PUF.

Corcuff, P. (1991). «Le catégoriel, le professionnel et la classe». Genèses, 3: 55-72.

Crozier, M. (1964). Le phénomène bureaucratique. Paris: Seuil.

Crozier, M. y Friedberg, E. (1977), Lacteur et le système. Paris: Seuil.

D'Iribarne, P. (1989). La logique de l'honneur. Gestion des entreprises et traditions nationales. Paris: Seuil.

D'Iribarne, P. (1992). «Misère et grandeur d'un modèle français d'entreprise», en R. Sainsaulieu, (ed.). Lentreprise, une affaire de société. Paris: Presses de la FNSP, pp. 254-266.

Friedberg, E. (1993). Le pouvoir et la règle. Dynamiques de l'action organisée. Paris: Seuil.

Lazega, E. (1994). «Analyse de réseaux et sociologie des organisations». Revue française de sociologie, XXXV: 293-320.

Lıu, M. (1981). «Technologie, organisation du travail et comportements des salariés». Revue française de sociologie, XXII: 205-211.

Mathieu, C. (1986). «Organisation et gestion de la production dans une unité d'emboutissage: de la sociologie du travail à celle de l'entreprise». Sociologie du travail, 3. 
Maurice, M. (1992). «Les sociologues et l'entreprise», en R. Sainsaulieu (ed.). L'entreprise, une affaire de société. Paris: Presses de la FNSP, pp. 303-331.

Maurice, M.; Sellier, F. y Silvestre, J.-J. (1982). Politique d'éducation et organisation industrielle en France et en Allemagne. Paris: PUF.

Ramanantsoa, B. (1992). «L'autonomie stratégique de l'entreprise», en R. SainsauLieu (ed.). L'entreprise, une affaire de société. Paris: Presses de la FNSP, pp. 131147.

Reynaud, E. (1982). «Identités collectives et changement social: les cultures collectives comme dynamiques d'action». Sociologie du travail, 2.

Ribeill, G. (1984). Les cheminots. Paris: La Découverte.

Sainsaulieu, R. (1985). L'identité au travail. Paris: Presses de la FNSP.

Sainsaulieu, R. (1987). Sociologie de l'organisation et de l'entreprise. Paris: Presses de la FNSP, pp. 206-209.

Sainsaulieu, R. (1992). L'entreprise, une affaire de société. Paris: Presses de Sciences Po.

Sainsaulieu, R. (1992). «Lautonomie des entreprises dans le champ social», en R. Sainsaulieu (ed.). L'entreprise, une affaire de société. Paris: Presses de la FNSP, pp. 119-130.

Sainsaulieu, R. y Segrestin, D. (1986). «Vers une théorie sociologique de l'entreprise». Sociologie du travail, 3: 335-352.

Tixier, P.-E. (1988). «Légitimité et modes de domination dans les organisations». Sociologie du travail, 4: 615-629.

Tripier, M. (1986). «Culture ouvrière et culture d'entreprise. A propos de la disparition de la PME en milieu rural». Sociologie du travail, 3: 373-386. 